Dominique JACQUES ${ }^{1}$

Guibien Cléophas ZeRBo

Pierre Soloviev ${ }^{2,3}$

Djingdia LOMPO ${ }^{2}$

${ }^{1}$ Direction générale Agriculture, Ressources naturelles

et Environnement

Avenue Prince de Liège, 15

5100 Jambes

Belgique

${ }^{2}$ Centre national de semences

forestières

01 BP 2682

Ouagadougou 01

Burkina Faso

${ }^{3}$ Association pour la promotion de l'éducation et de la formation à l'étranger 01 BP 6625

Ouagadougou 01

Burkina Faso

\title{
Acacia senegal au Burkina Faso : stratégie d’amélioration génétique
}

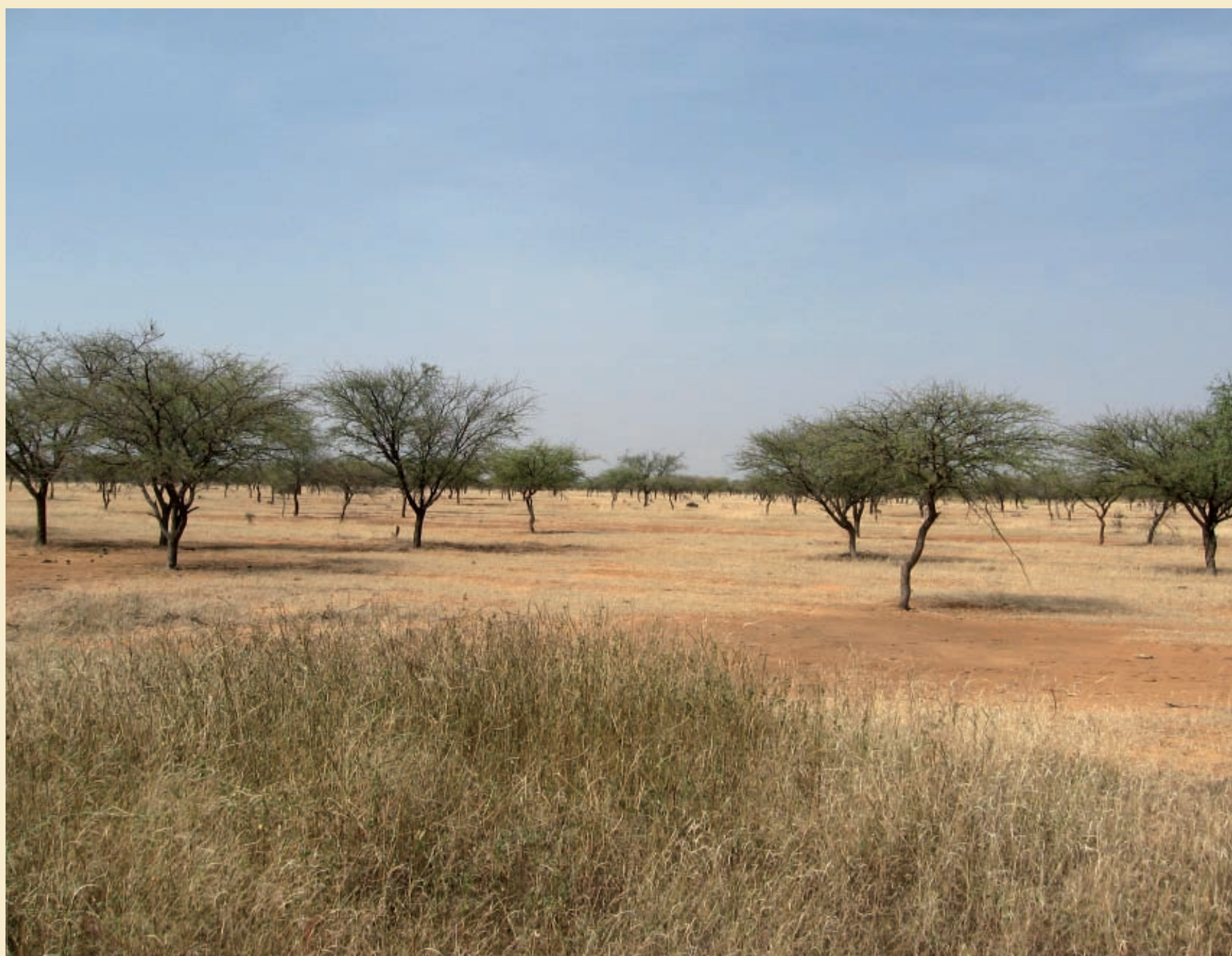

Photo 1.

Peuplement naturel d'Acacia senegal à Pacodé (province du Sanmatenga, Burkina Faso). Photo P. Soloviev. 


\section{RÉSUMÉ}

\section{ACACIA SENEGAL AU BURKINA FASO : STRATÉGIE D’AMÉLIORATION GÉNÉTIQUE}

Depuis près de dix ans, le Centre national de semences forestières développe un programme d'amélioration génétique d'Acacia senegal en vue d'accroître la production de gomme arabique au Burkina Faso. Différentes mesures pratiquées dans huit peuplements naturels ainsi que dans trois tests de provenances suivis pendant un à trois ans ont permis d'évaluer différents paramètres génétiques. Les résultats obtenus montrent une grande variabilité de la production au sein des provenances, qui se traduit par une héritabilité génotypique significative. La variabilité au niveau individuel apparaît encore plus élevée, la production des arbres les plus performants atteignant plus d'un kilo de gomme par an. Un effet " année » sur la production est également observé. Sur la base de ces résultats, les gains potentiels par sélection génétique sont estimés à partir de différents scénarios en prenant en compte la sélection au niveau des populations ainsi qu'au niveau individuel. La sélection individuelle semble être la plus efficace même si un gain significatif est aussi attendu par la sélection de peuplements à graines. Les dispositifs existants et en cours d'installation devraient permettre de poursuivre ce travail d'amélioration génétique aboutissant à la création de variétés améliorées. Enfin, le bon comportement de plusieurs provenances étrangères permet également d'entrevoir la possibilité d'élaborer un programme d'amélioration génétique à l'échelle régionale, couvrant une grande partie de l'Afrique sahélienne.

Mots-clés : Acacia senegal, amélioration génétique, gain génétique, héritabilité, gomme, Burkina Faso.

\section{ABSTRACT}

\section{ACACIA SENEGAL IN BURKINA FASO: A GENETIC IMPROVEMENT STRATEGY}

For almost ten years, The National Forest Seeds Centre has been developing a genetic improvement programme for Acacia senegal with a view to increasing Burkina Faso's gum arabic production. Different measurements in 8 natural stands and 3 provenance trials monitored over one to three years were used to assess various genetic parameters. The results show wide variability in production by provenance, reflected in significant genotype heritability. Individual variability is even higher, with the best trees producing over 1 kilogram of gum per year. A "year" effect on production was also observed. Based on these results, potential gains through genetic selection were estimated for different selection scenarios both at population and individual scale. Individual selection seems to be the most effective, but significant gains are also expected from selection of seed-tree populations. Work on genetic improvement to create improved varieties will continue through trials already under way or now becoming established. Finally, the promising behaviour of several foreign provenances suggests possibilities for a genetic improvement programme at regional scale covering large areas of the Sahel.

Keywords: Acacia senegal, genetic improvement, genetic gain, heritability, gum, Burkina Faso.

\section{RESUMEN}

\section{ACACIA SENEGAL EN BURKINA FASO: ESTRATEGIA DE MEJORA GENÉTICA}

Desde hace casi diez años, el Centre national de semences forestières desarrolla un programa de mejora genética de Acacia senegal para incrementar la producción de goma arábiga en Burkina Faso. Las diferentes mediciones efectuadas en ocho rodales naturales, así como en tres ensayos de procedencias monitoreados durante uno a tres años, permitieron evaluar distintos parámetros genéticos. Los resultados logrados muestran una gran variabilidad de la producción entre las procedencias, que se refleja en una heredabilidad genotípica significativa. La variabilidad individual es aún más elevada, la producción de los árboles con mayor desempeño superó el kilo de goma anual. También se observó un efecto "año" sobre la producción. Basándose en estos resultados, se estiman las potenciales ganancias de la selección genética a partir de distintos escenarios, teniendo en cuenta tanto la selección en poblaciones como a nivel individual. La selección individual parece ser la más eficaz, aunque también se espere una ganancia significativa mediante la selección de rodales semilleros. Los ensayos ya existentes o en proceso de instalación deberían permitir proseguir este trabajo de mejora genética y desembocar en a la creación de variedades mejoradas. Por último, el buen comportamiento de varias procedencias extranjeras permite también vislumbrar la posibilidad de elaborar un programa de mejoramiento genético a escala regional que abarque un amplio sector del África saheliana.

Palabras clave: Acacia senegal, mejora genética, ganancia genética, heredabilidad, goma, Burkina Faso. 


\section{Introduction}

Acacia senegal (L.) Willd. est un petit arbre connu mondialement pour ses nombreuses qualités, dont notamment son aptitude à produire de la gomme arabique (DEANS et al., 1999 ; ICKowicz et al., 2005 ; ARBonNIER, 2009). Depuis près de 10 ans, un effort soutenu a été produit par le Cnsf $^{1}$ au Burkina Faso pour mener un programme d'amélioration génétique appliqué à cette espèce, axé principalement sur la production de graines améliorées par la sélection de peuplements et la création de vergers (Soloviev et al., 2010). Au cours de cette période, un grand nombre de données portant sur la répartition spécifique et l'importance des ressources en Acacia gommiers, ainsi que sur le potentiel de production de gomme à l'échelle du pays, ont été collectées. SOLOViev et al. (2009) ont montré que les ressources disponibles en peuplements naturels d'A. senegal au Burkina Faso sont limitées et que l'espèce se rencontre essentiellement en peuplements mélangés avec d'autres espèces du genre Acacia comme A. laeta R. Br. ex-Benth. ou A. dudgeoni Craib ex-Holland (photo 1 ). Ces mêmes auteurs ont également mis en évidence le faible nombre de peuplements artificiels d'A. senegal, conduisant à constater que les ressources actuellement exploitables sont largement insuffisantes pour assurer le développement d'une filière de production de gomme arabique à large échelle (photo 2).

S'agissant de la production individuelle de gomme arabique, le potentiel observé au Burkina Faso apparaît également assez faible par rapport aux attentes des producteurs. BALIMA (2000) observe une faible productivité pour certains peuplements naturels, avoisinant une production annuelle de 35 grammes (g) de gomme par arbre pour les moins productifs. Plus récemment, SoLOVIEV et al. (2010) ont obtenu, pour un ensemble de huit peuplements naturels, une production annuelle moyenne par arbre de $117 \mathrm{~g}$, variant de $45 \mathrm{~g}$ à $225 \mathrm{~g}$ selon le peuplement (photo 3 ).

Le développement d'une filière de production de gomme arabique viable ne peut donc se concevoir, au Burkina Faso, qu'en optant pour un programme de plantations à grande échelle, établies à partir d'un matériel génétique amélioré quant à son potentiel de production de gomme.

Photo 2.
Actuellement, il faut constater que peu d'informations sont disponibles au sein de la littérature internationale sur les paramètres génétiques caractérisant cette production. D’après les ressources documentaires consultées, seul OUEDRAOGO (2001) a établi à ce jour une estimation de paramètres génotypiques à l'échelle des provenances pour la production de gomme d'A. senegal et ce sur la base d'un essai faiblement productif. Il y fait état d'une héritabilité génotypique de l'ordre de 0,56 et d'un gain génotypique conventionnel de $26,6 \%$ correspondant à une intensité de sélection de $38 \%$. Pour les descendances, KANANJI (1993) obtient une héritabilité sur les moyennes très élevée atteignant 0,87 , mais dont l'estimation est très probablement imprécise étant donné qu'elle est calculée sur la base d'un essai ne comportant que six éléments génétiques originaires de différentes localités du Kordofan, au Soudan. Enfin, RADDAD et LUUKKANEN (2006), évaluant un test établi sur terrain argileux et comparant des provenances issues de régions sableuses et de régions argileuses, mettent en évidence une aptitude plus grande à la production de gomme arabique des provenances issues des régions à sols argileux, traduisant une potentielle variation génétique des populations étudiées en fonction des types de sols sur lesquels elles se développent.

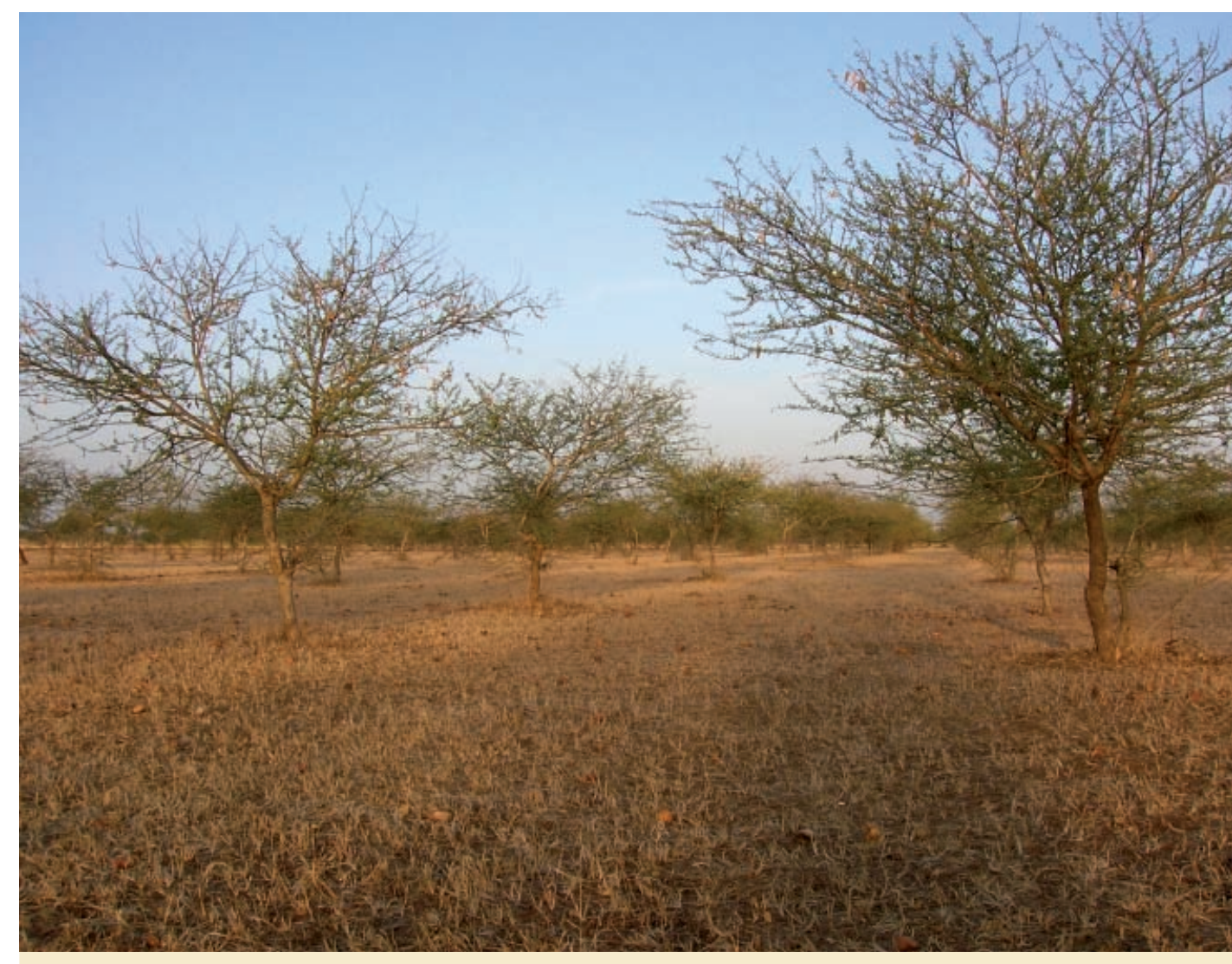

Peuplement artificiel d'Acacia senegal à Bogandé (province de la Gnagna, Burkina Faso). Photo P. Soloviev.

${ }^{1}$ Centre national de semences forestières. 


\section{Matériel et méthodes}

Les données utilisées proviennent d'essais de production de gomme réalisés dans huit peuplements naturels (tableau I) et trois essais de provenances (tableau II).

Au sein de chacun des huit peuplements naturels, répartis dans les trois régions de provenance couvrant l'aire de distribution de l'espèce au Burkina Faso, une sélection de 50 sujets adultes possédant un houppier équilibré et ne présentant pas de signes visibles de dépérissement ou d'attaques parasitaires et cryptogamiques a été opérée en veillant à leur répartition uniforme au sein du peuplement. Ces arbres ont ensuite été soumis durant deux ou trois années consécutives à des essais de production de gomme.

Parmi les essais de provenances, deux sont constitués de provenances locales ou étrangères parmi lesquelles sept à dix arbres ont été saignés au cours d'une ou de deux années. Ces arbres ont été sélectionnés sur la base des mêmes critères que ceux appliqués dans les peuplements naturels. Dans le troisième essai ne comportant qu'une seule provenance issue du Tchad, une saignée a été pratiquée sur 30 arbres choisis de manière aléatoire afin d'évaluer la production annuelle moyenne par arbre ainsi que la variabilité de cette caractéristique. Plus de détails sur ces différents essais et sur le mode opératoire sont fournis par SoLOVIEV et al. (2010).

La variable « production de gomme » ne présentant pas une distribution normale, une transformation $(\sqrt[3]{x})$ lui a été appliquée. Les valeurs des différents paramètres tels les coefficients de variation inter-peuplements et intra-peuplement pour l'ensemble des populations ainsi que l'héritabilité génotypique estimée dans deux des essais de provenances ont été calculées au départ de ces données. L'héritabilité génotypique sur les moyennes par provenance est définie comme la part de la variabilité globale observée entre les éléments génétiques, considérant que chacun de ces éléments est conservé identique à lui-même après reproduction (NANSON, 1970).

Le gain génotypique a également été déduit de ces résultats. Il correspond au gain, pour la caractéristique considérée, que l'on peut réaliser si l'on utilise les provenances sélectionnées dans les mêmes conditions environnementales et au même stade de développement.

Héritabilité et gain sont dérivés de l'analyse de la variance à un facteur en considérant la provenance comme un facteur aléatoire (NANSON, 2004).

Tableau II.

Localisation et caractéristiques des essais de provenances en stations expérimentales.

\begin{tabular}{|c|c|c|c|c|c|c|c|c|c|c|}
\hline Site & $\begin{array}{l}\text { Région de } \\
\text { provenance }\end{array}$ & Latitude & Longitude & $\begin{array}{l}\text { Altitude } \\
\text { (m) }\end{array}$ & $\begin{array}{l}\text { Pluviométrie } \\
\text { annuelle } \\
\text { moyenne } \\
(\mathrm{mm})\end{array}$ & $\begin{array}{l}\text { Superficie } \\
\text { (ha) }\end{array}$ & $\begin{array}{l}\text { Année de } \\
\text { plantation }\end{array}$ & $\begin{array}{c}\text { Nombre } \\
\text { d'arbres } \\
\text { testés }\end{array}$ & $\begin{array}{l}\text { Origine des } \\
\text { provenances }\end{array}$ & $\begin{array}{l}\text { Pluviométrie } \\
\text { annuelle } \\
\text { moyenne } \\
\text { des sites } \\
\text { d'origine }(\mathrm{mm})\end{array}$ \\
\hline \multirow[t]{4}{*}{ Djomga } & \multirow[t]{4}{*}{ Sahélienne } & \multirow[t]{4}{*}{ N 140.5’31" } & \multirow[t]{4}{*}{ W $00^{\circ} 02^{\prime} 24^{\prime \prime}$} & \multirow[t]{4}{*}{280} & \multirow[t]{4}{*}{$510^{\star}$} & \multirow[t]{4}{*}{0,34} & \multirow[t]{4}{*}{1988} & 10 & $\begin{array}{l}\text { Ranerou/ } \\
\text { Sénégal }\end{array}$ & $550^{\star \star}$ \\
\hline & & & & & & & & 9 & $\begin{array}{l}\text { Guidich/ } \\
\text { Sénégal }\end{array}$ & $500^{\star \star}$ \\
\hline & & & & & & & & 7 & $\begin{array}{l}\text { Diamenar/ } \\
\text { Sénégal }\end{array}$ & $400^{\star \star}$ \\
\hline & & & & & & & & 10 & $\begin{array}{l}\text { Velingara } \\
\text { Sénégal }\end{array}$ & $550^{\star \star}$ \\
\hline \multirow[t]{8}{*}{ Gonsé } & \multirow[t]{8}{*}{$\begin{array}{c}\text { Nord } \\
\text {-soudanienne }\end{array}$} & \multirow[t]{8}{*}{ N $12^{\circ} 21^{\prime} 31^{\prime \prime}$} & \multirow[t]{8}{*}{ W $01^{\circ} 18^{\prime} 55^{\prime \prime}$} & \multirow[t]{8}{*}{278} & \multirow[t]{8}{*}{$750^{\star}$} & \multirow[t]{8}{*}{0,95} & \multirow[t]{8}{*}{1988} & 10 & $\begin{array}{l}\text { Thiarène/ } \\
\text { Sénégal }\end{array}$ & $300^{\star \star}$ \\
\hline & & & & & & & & 10 & $\begin{array}{l}\text { Namarel/ } \\
\text { Sénégal }\end{array}$ & $332^{\star \star}$ \\
\hline & & & & & & & & 10 & $\begin{array}{l}\text { Diaguely/ } \\
\text { Sénégal }\end{array}$ & $400^{\star \star}$ \\
\hline & & & & & & & & 10 & $\begin{array}{l}\text { Gueye Kadar } \\
\text { Sénégal }\end{array}$ & $309^{\star \star}$ \\
\hline & & & & & & & & 10 & $\begin{array}{l}\text { Windou/ } \\
\text { Sénégal }\end{array}$ & $350^{\star \star}$ \\
\hline & & & & & & & & 10 & $\begin{array}{c}\text { Fallatu forest/ } \\
\text { Soudan }\end{array}$ & $365^{\star \star}$ \\
\hline & & & & & & & & 10 & $\begin{array}{c}\text { Lac Dem/ } \\
\text { Burkina Faso }\end{array}$ & $750^{\star \star}$ \\
\hline & & & & & & & & 10 & $\begin{array}{c}\text { Boron/ } \\
\text { Burkina Faso }\end{array}$ & $900 * \star$ \\
\hline Gonsé & $\begin{array}{c}\text { Nord } \\
\text {-soudanienne }\end{array}$ & N 12²1’31" & W $01^{\circ} 18^{\prime} 55^{\prime \prime}$ & 278 & $750^{\star}$ & 1,11 & 1997 & 30 & $\begin{array}{l}\text { Bou Loulou } \\
\text { /Tchad }\end{array}$ & $500^{\star \star \star}$ \\
\hline
\end{tabular}




\section{Résultats et discussion}

\section{Héritabilité génotypique}

L'analyse des tests de provenances de Djomga et de Gonsé fournit des résultats très contrastés. Pour le site de Djomga regroupant quatre peuplements originaires du Sénégal, les résultats de l'analyse de la variance apparaissent très hautement significatifs et l'héritabilité génotypique qui en est dérivée atteint 0,89 , traduisant un très grand effet génétique sur la production de gomme. À l'opposé, l'analyse des données du test de provenance de Gonsé ne montre pas d'effet « provenance » significatif. Ce second résultat contraste avec celui obtenu, pour ce même essai, par OUEDRAOGo en 2001 qui fait état d’une héritabilité génotypique de 0,56. La plus grande puissance du test suite au nombre plus important de données collectées $(n=362)$ ainsi que l'âge moins avancé des plantes lors de cette précédente évaluation pourraient expliquer cette différence d'héritabilité. La faible production moyenne pourrait aussi avoir limité l'expression de la variabilité réelle de ces différentes provenances.

Sur la base de ces résultats, il est donc difficile d'évaluer avec précision le niveau d'héritabilité potentiel au niveau interpeuplements pour cette caractéristique. Cependant, les résultats enregistrés jusqu'à présent au Burkina Faso tendent à montrer l'existence d'un effet génétique significatif sur la production de gomme et confortent ceux repris dans les rares références recensées dans la littérature sur ce sujet (KANANJı, 1993).

\section{Variabilité de la production de gomme}

\section{Variabilité inter-peuplements}

Tant les données récoltées dans les peuplements naturels que celles issues des deux essais de provenances indiquent une grande variabilité de la production de gomme arabique entre les peuplements testés (tableau III).

Sur ces trois groupes évalués, les coefficients de variation sont relativement proches et compris entre 50 et $60 \%$, que ce soit pour les peuplements naturels ou pour les deux tests de comparaison de provenances. Dans les peuplements in situ, les conditions de site ainsi que les différences d'âge des arbres soumis aux tests de production de gomme ne semblent pas constituer des sources essentielles de variabilité de la production.

Tableau III.

Évaluation de la variabilité inter-peuplements de la production individuelle moyenne annuelle de gomme (en g) dans différents sites au Burkina Faso et estimation de la différentielle de sélection métrique (en g) pour une intensité de sélection de $25 \%$.

Site

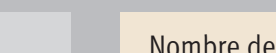
peuplements

Djomga Gonsé Peuplements

Moyenne
(g)

123,6

17,3

116,4

\begin{tabular}{|c|c|c|}
$\begin{array}{l}\text { CV } \\
(\%)\end{array}$ & Min & Max \\
\hline 54 & 39 & 168 \\
\hline 57 & 5 & 33 \\
\hline 50 & 46 & 226 \\
\hline
\end{tabular}

Différentielle de sélection métrique (g)

52

14

81

\section{Variabilité intra-peuplement}

Les coefficients de variation calculés au niveau individuel s'avèrent encore plus élevés qu'au niveau des peuplements (tableau IV).

En moyenne, le coefficient de variation dans les huit peuplements naturels est de l'ordre de $85 \%$ et compris entre $54 \%$ et $112 \%$. L'amplitude des résultats observée pour ces peuplements est également très importante; dans les trois peuplements les plus productifs, cette amplitude de production moyenne calculée sur deux ou trois ans atteint environ $750 \mathrm{~g}$ et, si l'on s'intéresse à la production d'une seule année, elle culmine à près de $1300 \mathrm{~g}$.

Cet ensemble de données confirme l'existence d'une grande variabilité de cette caractéristique au Burkina Faso, situation qui semble également exister dans les populations d'autres pays (BALLAL et al., 2005 ; RADDAD et LUUKKANEN, 2006). Le calcul de ces coefficients de variation dans l'essai de provenances de Djomga (tableau V) donne des chiffres variant entre $38 \%$ et $112 \%$ pour les quatre populations provenant du Sénégal, mais ces données doivent être interprétées avec précaution étant donné le faible nombre d'arbres par provenance pris en compte. Le coefficient de variation atteint $174 \%$ pour la provenance du Tchad installée à Gonsé, pour laquelle 30 arbres ont été étudiés. Il faut cependant noter que, probablement, ces deux derniers résultats obtenus sur une seule saison de récolte de gomme sont légèrement surestimés par rapport à ceux obtenus dans les huit peuplements naturels puisque, dans ce dernier cas, les coefficients sont calculés à partir des moyennes de production de gomme établies sur deux ou trois ans. Cette grande variabilité est aussi observée par RAEBILD et al. (2003), dans des essais de provenances établis au Burkina Faso, pour les caractéristiques de croissance. Ce constat permet par ailleurs d'envisager un programme d'amélioration génétique combinant plusieurs caractéristiques.

\section{Gains potentiels par sélection génétique}

À partir des données collectées, il est possible d'évaluer les gains génotypiques qui pourraient être obtenus par sélection d'une partie des peuplements étudiés et par sélection individuelle au travers de différents scénarios fixant, d'une part, une intensité de sélection des éléments génétiques et, d'autre part, différents niveaux d’héritabilité.

\section{Sélection des peuplements}

Connaissant le coefficient de variation phénotypique, il est possible d'évaluer le gain génotypique de production en gomme qui pourrait être obtenu par l'utilisation d'une partie des peuplements les plus performants pour la récolte de graines.

Ce gain dépend du niveau d'héritabilité génotypique de cette caractéristique ainsi que de l'intensité de sélection retenue pour effectuer cette sélection. Sur la base d'un coefficient de variation phénotypique de $50 \%$, estimé à partir de l'analyse de la variance du test de Djomga et des valeurs observées pour les différents sites naturels, la figure 1 présente la gamme de gains possibles. 


\section{Tableau IV.}

Évaluation de la production annuelle moyenne de gomme par arbre (en g), de différents paramètres statistiques (coefficient de variation, minimum et maximum) et de la différentielle de sélection métrique pour une intensité de sélection variant de 2 à $25 \%$ pour différentes populations d'Acacia senegal mesurées au cours d'une à trois années.

\begin{tabular}{|c|c|c|c|c|c|c|c|c|c|c|c|}
\hline \multirow[t]{2}{*}{ Site } & \multirow[t]{2}{*}{ Effectif } & \multirow[t]{2}{*}{$\begin{array}{l}\text { Nombre } \\
\text { d'années }\end{array}$} & \multirow[t]{2}{*}{$\begin{array}{c}\text { Moyenne } \\
\text { (g) }\end{array}$} & \multirow[t]{2}{*}{$\begin{array}{l}\text { Écart- } \\
\text { type }\end{array}$} & \multirow[t]{2}{*}{$\begin{array}{l}\text { CV } \\
(\%)\end{array}$} & \multirow[t]{2}{*}{ Min } & \multirow[t]{2}{*}{ Max } & \multicolumn{4}{|c|}{$\begin{array}{l}\text { Différentielle de sélection métrique } \\
\text { pour différentes intensités de sélection (g) }\end{array}$} \\
\hline & & & & & & & & $25 \%$ & $10 \%$ & $6 \%$ & $2 \%$ \\
\hline Bissiga & 50 & 3 & 226 & 188 & 83 & 23 & 803 & 262 & 438 & 515 & 577 \\
\hline Konladé & 50 & 3 & 104 & 56 & 54 & 20 & 241 & 77 & 108 & 118 & 138 \\
\hline Fènèguènè & 50 & 3 & 90 & 101 & 112 & 0 & 449 & 98 & 172 & 208 & 261 \\
\hline Doussoula & 50 & 2 & 72 & 55 & 76 & 0 & 232 & 73 & 104 & 121 & 161 \\
\hline Pacodé & 50 & 2 & 46 & 30 & 66 & 0 & 103 & 41 & 51 & 55 & 58 \\
\hline Soumdiguidi & 50 & 2 & 158 & 141 & 89 & 14 & 839 & 180 & 308 & 377 & 681 \\
\hline Yeimzuro & 50 & 2 & 169 & 154 & 92 & 0 & 599 & 212 & 326 & 400 & 430 \\
\hline $\begin{array}{l}\text { Gonsé } \\
\text { (prov. Tchad) }\end{array}$ & 30 & 1 & 109 & 190 & 174 & 0 & 859 & 244 & 464 & 536 & 750 \\
\hline
\end{tabular}

Il apparaît que, si 38 \% des meilleurs peuplements sont retenus, une amélioration de la production de l'ordre de 20 à $35 \%$ peut être espérée, ce qui représenterait un gain en production d'un maximum de $41 \mathrm{~g}$ si l'on appliquait cette sélection aux huit peuplements naturels de cet essai. Si l'on passe, par contre, à un taux de sélection de $10 \%$, le gain en production moyenne annuelle par arbre pourrait atteindre 35 à $61 \%$, soit un gain maximum de $71 \mathrm{~g}$; les plantations issues de ce matériel génétique pourraient atteindre alors une production annuelle moyenne par arbre d'environ $187 \mathrm{~g}$. Il est théoriquement possible d'appliquer des taux de sélection plus importants tels que présentés dans cette même figure mais, pratiquement, l'investissement humain et financier nécessaire à l'évaluation d'un grand nombre de peuplements risquerait d'être alors trop important par rapport au gain supplémentaire attendu.

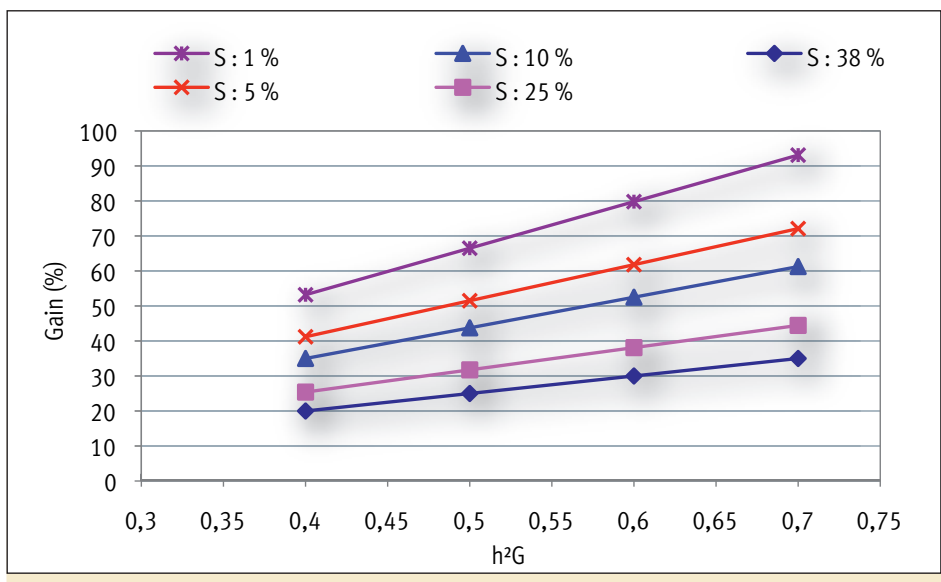

Figure 1.

Évaluation du gain génotypique escompté exprimé en pourcentage de la moyenne pour la production de gomme par sélection de peuplements au Burkina Faso pour différents niveaux d'héritabilité génotypique $\left(h^{2} G\right)$ et différentes différentielles de sélection (S).

\section{Tableau V. \\ Évaluation de la variabilité individuelle de la production annuelle moyenne de gomme (en g) au sein de différentes provenances étrangères testées au Burkina Faso.}

\begin{tabular}{|c|c|c|c|c|c|c|c|c|}
\hline Site d'essai & Provenance & Origine & $\begin{array}{c}\text { Nombre } \\
\text { d'arbres testés }\end{array}$ & $\begin{array}{l}\text { Moyenne } \\
\text { (g) }\end{array}$ & Écart-type & CV (\%) & Min & Max \\
\hline \multirow[t]{4}{*}{ Djomga } & Ranerou & Sénégal & 10 & 168 & 64 & 38 & 38 & 260 \\
\hline & Guidich & Sénégal & 9 & 90 & 56 & 61 & 34 & 200 \\
\hline & Diamenar & Sénégal & 7 & 39 & 44 & 112 & 0 & 102 \\
\hline & Velingara & Sénégal & 10 & 168 & 104 & 62 & 34 & 371 \\
\hline Gonsé & Bou Loulou & Tchad & 30 & 109 & 190 & 174 & 0 & 859 \\
\hline
\end{tabular}




\section{Sélection individuelle}

Le coefficient de variation moyen intra-peuplement étant supérieur au coefficient de variation inter-peuplements, la sélection au niveau individuel est potentiellement plus efficace que celle des peuplements, tout autre facteur restant constant (tableau VI). À titre d'exemple, pour une héritabilité supposée de 0,5 et une intensité de sélection de $25 \%$, le gain estimé par sélection des peuplements atteint $32 \%$, alors que celui obtenu par sélection individuelle est de $59 \%$, soit un accroissement de $85 \%$. La supériorité de ce type de sélection peut aussi être renforcée en appliquant une intensité de sélection plus forte pour un même niveau d'investissement. Dans cette hypothèse, pour des intensités de sélection variant de 2 à $25 \%$ et suivant le niveau d'héritabilité escompté, des gains de l'ordre de 100 à $200 \%$ de la moyenne de production peuvent être attendus. La production moyenne par individu des plantations issues de ce matériel amélioré pourrait alors atteindre 240 à $350 \mathrm{~g}$. Si l'on envisage des plantations à des écartements de $5 \mathrm{~m}$ sur $5 \mathrm{~m}$, en tenant compte d'un taux de survie de $85 \%$, une production annuelle moyenne de gomme de l'ordre de 80 à $120 \mathrm{~kg}$ par hectare pourrait être atteinte. Ces estimations restent cependant entachées d'une erreur difficilement estimable puisqu'il n'est pas possible d'évaluer l'héritabilité génétique au sens strict de ce caractère à partir des données disponibles actuellement, même si quelques résultats tendant à l'établir sont publiés pour cette espèce (KANANJI, 1993) ou pour d'autres espèces productrices d'exsudats (SQUILLACE, BENGTSON, 1961 ; ALIKA, 1985). Il convient donc de rester prudent pour l'exploitation pratique de ces résultats.

\section{Effet année}

Le tableau VII, présentant les productions en gomme des dix meilleurs arbres producteurs parmi les quatre peuplements dont la production de gomme arabique a été suivie pendant trois années successives, montre qu'il existe une assez grande variabilité annuelle pour cette caractéristique. Elle se traduit par un coefficient de variation compris entre 19 et $72 \%$ et une différence annuelle maximale de production atteignant $1091 \mathrm{~g}$ pour l'arbre numéro 2. Ces observations pourraient donc justifier des mesures répétées sur plusieurs années dans le cadre d'une sélection d' « arbres plus », même si les trois coefficients de corrélation calculés entre ces différentes séries de données pour ces quatre peuplements sont très hautement significatifs, compris entre 0,62 et 0,65 . La répétition de ces mesures a cependant un surcoût qui pourrait être utilisé pour augmenter le nombre d'arbres évalués, ce qui permettrait d'accroître l'intensité de sélection et d'augmenter potentiellement le gain final. Pour vérifier cette hypothèse, une simulation a été réalisée à partir des 200 arbres dont la production annuelle de gomme a été mesurée durant trois années consécutives. En supposant des moyens financiers et humains disponibles permettant d'effectuer la saignée d'un total de 600 arbres, répartis de trois manières, 200 arbres testés durant trois années, 300 arbres testés durant deux années ou 600 arbres testés sur une seule année, cela donne, pour une sélection finale de 30 arbres, une intensité de sélection de respectivement 15,10 et $5 \%$. Les résultats montrent que la moyenne de production des 30 meilleurs arbres sélectionnés sur les 200 arbres suivis durant trois années s'établit à $369 \mathrm{~g}$ et correspond à la production la plus faible. En revanche, les 30 meilleurs arbres

\begin{abstract}
Tableau VI.
Évaluation du gain génotypique escompté exprimé en pourcentage de la production moyenne annuelle de gomme par sélection individuelle au Burkina Faso pour différents niveaux d'héritabilité génotypique et différentes intensités de sélection.
\end{abstract}

\begin{tabular}{l|c|}
\hline Site & $\begin{array}{c}\text { Moyenne } \\
(\mathrm{g})\end{array}$ \\
& \\
\hline Bissiga & \\
\hline Konladé & 104 \\
\hline Fènèguènè & 90 \\
\hline Guitalé & 67 \\
\hline Doussoula & 72 \\
\hline Pacodé & 46 \\
\hline Soumdiguidi & 158 \\
\hline Yeimzuro & 169 \\
\hline Moyenne & 116 \\
\hline Gonsé & 109 \\
\hline prov. Tchad) & \\
\hline
\end{tabular}

0,3

\begin{tabular}{|c|c|c|}
\hline $25 \%$ & $10 \%$ & $6 \%$ \\
\hline 35 & 58 & 68 \\
\hline 22 & 31 & 34 \\
\hline 32 & 57 & 69 \\
\hline 64 & 110 & 130 \\
\hline 31 & 44 & 51 \\
\hline 27 & 33 & 36 \\
\hline 34 & 58 & 71 \\
\hline 38 & 58 & 71 \\
\hline 35 & 56 & 66 \\
\hline 67 & 128 & 148 \\
\hline
\end{tabular}

$h^{2} \mathrm{G}$

0,5 Intensité de sélection $25 \% \quad 10 \% \quad 6 \% \quad 2 \%$

$\begin{array}{rrrr}58 & 97 & 114 & 128 \\ 37 & 52 & 57 & 66 \\ 54 & 95 & 115 & 144 \\ 107 & 184 & 217 & 269 \\ 51 & 73 & 84 & 112 \\ 45 & 56 & 60 & 63 \\ 57 & 97 & 119 & 215 \\ 63 & 97 & 119 & 128 \\ 59 & 94 & 111 & 141 \\ 112 & 213 & 246 & \\ & & & \end{array}$

0,7

\begin{tabular}{rrrr}
$25 \%$ & $10 \%$ & $6 \%$ & $2 \%$ \\
81 & 136 & 159 & 179 \\
\hline 52 & 73 & 80 & 93 \\
76 & 133 & 161 & 202 \\
\hline 150 & 258 & 304 & 377 \\
\hline 71 & 102 & 118 & 157 \\
\hline 63 & 78 & 84 & 89 \\
\hline 80 & 136 & 167 & 301 \\
\hline 88 & 135 & 166 & 179 \\
83 & 131 & 155 & 197 \\
\hline 157 & 298 & 344 & \\
\hline
\end{tabular}


Tableau VII.

Productions annuelles et moyennes de gomme (en g) des 10 arbres les plus performants.

\begin{tabular}{|c|c|c|c|c|c|}
\hline$N^{\circ}$ ordre & & ctions annuel & & Moyenne (g) & CV (\%) \\
\hline & 2006-2007 & 2007-2008 & 2008-2009 & & \\
\hline 1 & 718,5 & 1009,2 & 681,4 & 803,0 & 22 \\
\hline 2 & 942,7 & 1262,7 & 171,8 & 792,4 & 71 \\
\hline 3 & 922,2 & 384,5 & 576,1 & 627,6 & 43 \\
\hline 4 & 376,9 & 593,5 & 686,5 & 552,3 & 29 \\
\hline 5 & 459,4 & 494,5 & 688,2 & 547,4 & 23 \\
\hline 6 & 579,0 & 621,6 & 281,7 & 494,1 & 37 \\
\hline 7 & 525,9 & 265,9 & 592,1 & 461,3 & 37 \\
\hline 8 & 188,4 & 810,7 & 347,0 & 448,7 & 72 \\
\hline 9 & 368,8 & 389,5 & 522,0 & 426,8 & 19 \\
\hline 10 & 479,8 & 368,1 & 281,4 & 376,4 & 26 \\
\hline
\end{tabular}

\section{Valorisation des premiers résultats}

Ces premiers résultats permettent de préciser et d'optimiser les opérations de récolte de graines pour cette espèce. L'existence d'une héritabilité génotypique significative au niveau des peuplements confirme l'intérêt de sélectionner, comme peuplements destinés à la récolte de graines, les populations qui ont montré une production supérieure de gomme arabique au cours de deux à trois ans d'évaluation. Les gains espérés ne seront cependant pas très élevés et ne devraient pas dépasser quelques dizaines de grammes de gomme par arbre et par année dans les conditions du Burkina Faso, mais avec l'avantage d'être accessibles dès à présent. Ces gains pourraient être quelque peu accrus en pratiquant des éclaircies sélectives destinées à supprimer les individus les moins productifs. Mais, comme le signalent SoLoviev et al. (2010), ces opérations doivent être entreprises avec précaution, afin d'éviter

sur les 600 retenus sur la base d'une seule année de saignée atteindraient une production moyenne de $489 \mathrm{~g}$, soit un gain de $33 \%$; la sélection opérée sur les 300 arbres testés durant deux années de suivi donnerait un résultat intermédiaire de $411 \mathrm{~g}$. Ces résultats tendent donc à montrer que la sélection d'un grand nombre d'individus mesurés une seule année est plus efficace en termes de gain potentiel que la sélection d'un plus petit nombre d'arbres suivis pendant deux ou trois années successives.

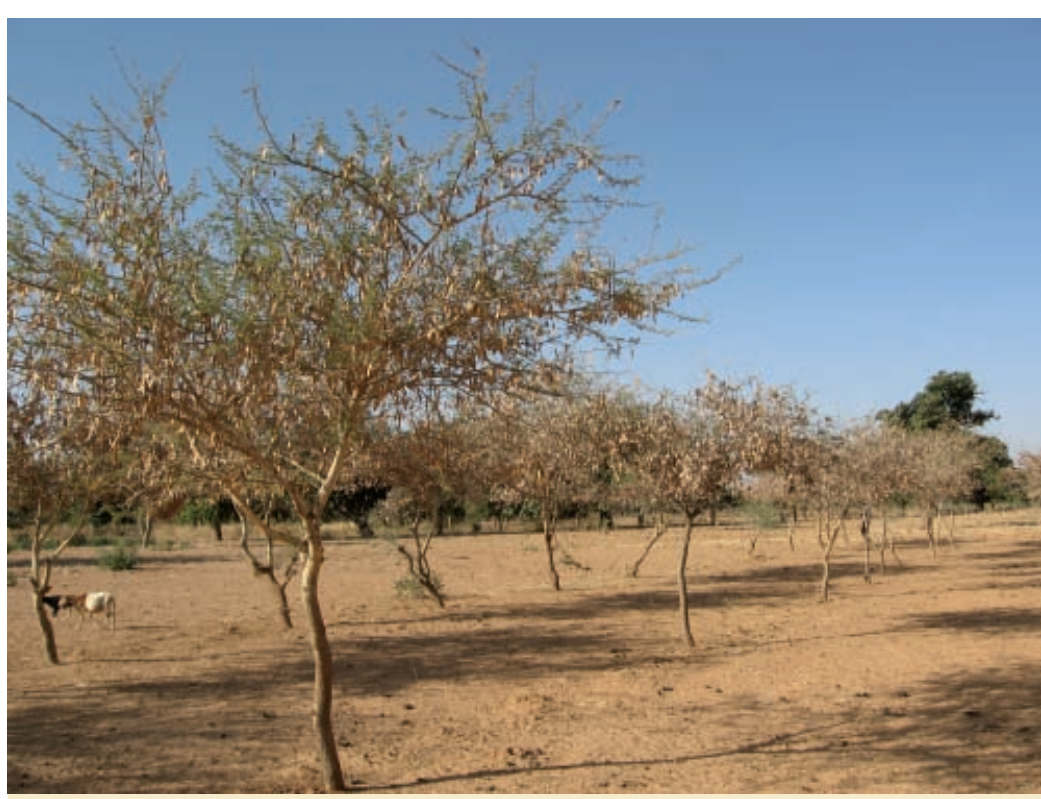

Photo 4.

Fructification abondante dans un peuplement artificiel d'Acacia senegal à Ziré (province du Zondoma, Burkina Faso).

Photo P. Soloviev. une perception négative de ces éclaircies sélectives par les populations locales avoisinantes. Une autre solution, n'impliquant pas l'élimination des individus les moins productifs, consiste à effectuer les récoltes uniquement sur les meilleurs individus, mais dans ces conditions le gain de production attendu à la génération suivante dans les peuplements issus de ces individus librement pollinisés sera réduit théoriquement de moitié, suite au croisement de ces bons producteurs avec des individus moins performants (NANSON, 2004). Les différents lots de graines ainsi récoltés pourraient alors être regroupés par région de provenance (CNSF, 2008). En considérant un potentiel de production de graines qui peut atteindre, dans des conditions normales, plus de $500 \mathrm{~g}$ de graines par arbre, les besoins annuels en graines du Burkina Faso qui s'élèvent à environ $100 \mathrm{~kg}$ à l'heure actuelle pourraient facilement être couverts dès à présent par ce type de récolte ciblée (photo 4).

Les résultats obtenus confirment, d'autre part, la pertinence de la stratégie d'amélioration génétique suivie pour cette espèce, par la création de vergers à graines au départ des arbres les plus performants. Toutefois, dans ce cas, les premières graines ne seront obtenues qu'à moyen terme, c'est-à-dire d'ici cinq à huit ans (photo 5). Compte tenu de l'existence d'un gain génotypique potentiel par sélection des meilleurs peuplements, les vergers à graines devraient inclure une proportion plus importante des arbres issus de ces meilleurs peuplements, tout en veillant à assurer une large diversité génétique à ces matériels de base. 


\section{Conclusion et perspectives}

Pour bénéficier au plus tôt du travail de sélection des « arbres plus » et compte tenu de la très bonne aptitude au greffage de cette espèce dans les conditions du Burkina Faso, la création d'une variété multiclonale pourrait également être envisagée. Afin d'assurer une diversité génétique minimale dans chacune des plantations et de garder l'opportunité d'opter pour la régénération naturelle pour les générations ultérieures, un minimum d'une trentaine de clones devrait composer cette variété. Cette option suppose cependant l'absence d'incompatibilité de greffage à long terme et un effet limité des porte-greffes sur l'aptitude à la production de gomme des clones greffés, éléments mal maîtrisés dans l'état actuel des connaissances. L'utilisation de boutures, autre technique de multiplication végétative applicable à $A$. senegal (N'DIAYE et al., 1991 ) mais non encore expérimentée au Cnsf, pourrait constituer une alternative encore plus intéressante.

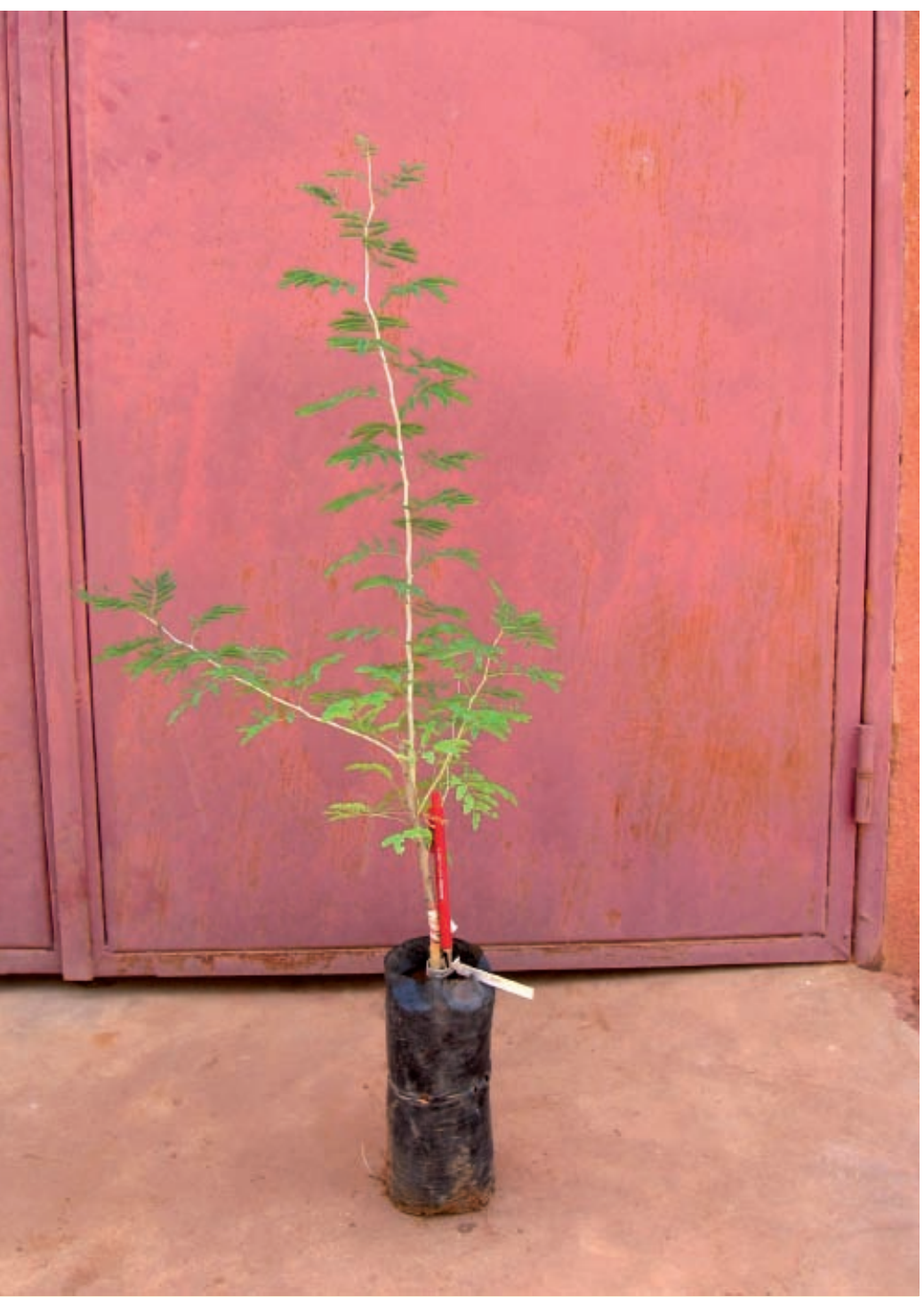

Photo 5.

Ramet d'un « arbre plus » d'Acacia senegal obtenu par greffage à l'antenne du Cnsf à Kaya (Burkina Faso). Photo D. Jacques.
Au Burkina Faso, les essais menés au Cnsf depuis une dizaine d'années dans le cadre de la conduite d'un programme d'amélioration génétique de l'espèce $A$. senegal montrent l'existence d'une grande variabilité de la production de gomme arabique. Celle-ci est observée tant au niveau inter-peuplements qu'intra-peuplement, aussi bien pour les peuplements naturels du Burkina Faso que pour les populations issues de plusieurs pays sahéliens, comme le Sénégal et le Tchad, présentes dans les tests de provenances mis en place. Les résultats obtenus sur les niveaux d'héritabilité, même s'ils sont encore partiels, tendent à montrer qu'il est possible de développer un programme de sélection de matériels performants au travers de la sélection de peuplements et de la sélection individuelle.

Les essais mis en place et les sélections réalisées jusqu'à présent au Burkina Faso permettent d'envisager la poursuite des travaux d'amélioration génétique. Les vergers installés offrent la possibilité d'évaluer la production de gomme des clones sélectionnés dans des conditions contrôlées, ce qui fournira une estimation de l'héritabilité génotypique au niveau clonal. Une estimation plus précise des paramètres génétiques liés à la production de gomme pourrait également être établie grâce à l'installation d'un test de descendances. Cet ensemble de tests pourrait déboucher sur la mise en place d'un verger d'élite produisant des graines de la catégorie «testée » selon le système $\mathrm{Ocde}^{2}$, lorsque celleci aura statué sur cette catégorie.

Les bons résultats obtenus par les provenances étrangères en termes de production de gomme suggèrent qu'il serait envisageable de développer des programmes d'amélioration génétique communs à plusieurs pays d'Afrique de l'Ouest voire à l'échelle d'une grande partie de la zone sahélienne.

La mise en commun des moyens pourrait permettre d'intensifier les sélections et d'étendre celles-ci à d'autres caractéristiques telles que la production de matière ligneuse ou de fourrage (feuilles et gousses), voire l'aptitude à la fixation d'azote dans le cadre de la restauration de la fertilité des sols ou dans la perspective d'une intégration d'A. senegal dans un système agroforestier.

\section{Remerciements}

Ce travail a pu être mené à bien grâce au soutien de l'Association pour la promotion de l'éducation et de la formation à l'étranger (Apefe), projet intitulé « Renforcement structurel du Cnsf en vue de développer la filière gomme arabique à travers la production de matériels forestiers de reproduction de qualité et la formation des producteurs », et de la Région wallonne de Belgique, projet intitulé «Valorisation de la gomme arabique - De la semence au produit transformé (phase finale) ».

${ }^{2}$ Organisation de coopération et de développement économiques. 


\section{Références bibliographiques}

ALIKA J. E., 1985. Heritability and genetic gain from selection in rubber (Hevea brasiliensis). Silvae Genetica, 34 (1) : 1-4. ARBONNIER M., 2009. Arbres, arbustes et lianes des zones sèches d'Afrique de l'Ouest. Versailles, France, Éditions Quæ, $574 \mathrm{p}$.

BADJI S., N'DIAYE I., DANTHU P., COLONNA J. P., 1991. Vegetative propagation studies of gum arabic trees. 1. Propagation of Acacia senegal (L.) Willd. using lignified cuttings of small diameter with eight nodes. Agroforestry Systems, $14: 183-191$.

BALIMA R., 2000. Essai de production de gomme arabique dans les peuplements naturels de Acacia spp. Ouagadougou, Burkina Faso, Centre national de semences forestières, Rapport technique $\mathrm{n}^{\circ} 31,43 \mathrm{p}$.

BALLAL M. E., EL SIDDIG E. A., ELFADL M. A., LUUKKANEN O., 2005. Relationship between environmental factors, tapping dates, tapping intensity and gum arabic yield of an Acacia senegal plantation in western Sudan. Journal of Arid Environments, 63 (2) : 379-389.

CNSF, 2008. Régions de provenance des espèces forestières et agroforestières du Burkina Faso. Ouagadougou, Burkina Faso, ministère de l'Environnement et du Cadre de Vie, Secrétariat général, Centre national de semences forestières, $19 \mathrm{p}$.

DANTHU P., LEBLANC J. M., BADJI S., COLONNA J. P., 1992. Vegetative propagation studies of gum arabic trees. 2 . The vegetative propagation of adult Acacia senegal. Agroforestry Systems, $19: 15-25$.

DANTHU P., SALL P. N., SECK M., 1998. Compatibilité de greffage entre quelques acacias africains. Premiers résultats. Bois et Forêts des Tropiques, 258 : 49-57.

DEANS J. D., DIAGNE O., LINDLEY D. K., DIONE M., PARKINSON J. A., 1999. Nutrient and organic-matter accumulation in Acacia senegal fallows over 18 years. Forest Ecology and Management, 124 (2-3) : 153-167.

ICKOWICZ A., FRIOT D., GUERIN H., 2005. Acacia senegal, arbre fourrager sahélien ? Bois et Forêts des Tropiques, 284 : 59-68.

KANANJI B., 1993. Variation in gum arabic production of six Sudanese Acacia senegal seed sources. In : Tree seed problems with special reference to Africa. Proceedings of the IUFRO Symposium, Ouagadougou, Burkina Faso. Leiden, Pays-Bas, Backhuys, p. 118-127.

NANSON A., 1970. L'héritabilité et le gain d'origine génétique dans quelques types d'expériences. Silvae Genetica, 19 : 113-121.

NANSON A., 2004. Génétique et amélioration des arbres forestiers. Gembloux, Belgique, Les Presses Agronomiques de Gembloux, 712 p.
N’DIAYE I., BADJI S., GESLOT A., MERLIN G., NEVILLE P., 1991. Recherche de conditions favorables à l'enracinement de boutures d'Acacia senegal (L.) Willd. In : Physiologie des arbres et arbustes en zones arides et semi-arides. Paris, France, Groupe d'étude de l'arbre, p. 315-322.

OUEDRAOGO M., 2001. Analyse statistique dans le cadre de l'amélioration génétique forestière en zone soudano-sahélienne : cas d'un essai de provenances d'Acacia senegal. Mémoire de fin d'études, Dea en Statistique et informatique appliquées, Faculté universitaire des sciences agronomiques de Gembloux, Belgique, 97 p.

RADDAD E. A. Y., LUUKKANEN O., 2006. Adaptative genetic variation in water-use efficiency and gum yield in Acacia senegal provenances grown on clay soil in the Blue Nile region, Sudan. Forest Ecology and Management, 226 : 219-229.

RAEBILD A., GRAUDAL L., OUEDRAOGO L. G., 2003. Evaluation of a provenance trial of Acacia senegal at Gonsé, Burkina Faso. Trial $n^{\circ} 12$ in the Arid Zones Series. Humlebaeck, Danemark, Danida Forest Seed Centre, Results and Documentation $\mathrm{n}^{\circ} 5,34 \mathrm{p}$.

SOLOVIEV P., ZERBO G. C., LOMPO D., YODA L. B., JACQUES D., DIALLO A., 2009. Acacia senegal au Burkina Faso : état de la ressource et potentiel productif. Bois et Forêts des Tropiques, $300: 15-25$.

SOLOVIEV P., JACQUES D., ZERBO G. C., LOMPO D., 2010. Production de semences améliorées d'Acacia senegal. Bois et Forêts des Tropiques, $303:$ 67-82.

SQUILLACE A. E., BENGTSON G. W., 1961. Inheritance of gum yield and other characteristics of slash pine. Proc. $6^{\text {th }}$ Southern Forest Tree Improvement Conference, Gainesville, Florida, USA, p. 85-96.

VASSAL J., 1991. État des connaissances sur l'induction de gommose chez Acacia senegal. In : Physiologie des arbres et arbustes en zones arides et semi-arides. Paris, France, Groupe d'étude de l'arbre, p. 271-276.

VASSAL J., SALL P. N., DIONE M., FENYO J.-C., VANDEVELDE M.-C., SERVANT-DUVALLET S., CHAPPUIS A., 1992. Modélisation du comportement de populations artificielles d'acacias gommiers (Acacia senegal) dans le Ferlo sénégalais. Compte rendu de fin de programme MRT $68 \mathrm{~L} 0465$, France, $75 \mathrm{p}$.

ZERBO G. C., 2005. Étude préliminaire de la sélection d' « arbres plus » de Acacia senegal (L.) Willd. : production de gomme et multiplication végétative par greffage. Travail de fin d'études, Université polytechnique de Bobo-Dioulasso, Burkina Faso, 66 p. 